\title{
Novel concepts for the chemoprevention of breast cancer through aromatase inhibition
}

\author{
Anne Kendall and Mitch Dowsett \\ Academic Department of Biochemistry, Wallace Wing, Royal Marsden NHS Foundation Trust, Fulham Road, London SW3 6JJ, UK \\ (Requests for offprints should be addressed to A Kendall; Email: anne.kendall@icr.ac.uk)
}

\begin{abstract}
Aromatase inhibitors (Als) have a proven role in the treatment of early and metastatic breast cancer. The success of tamoxifen in reducing the relative risk of developing hormone-sensitive breast cancer in chemoprevention trials has been hampered by their long-term toxicity profile. Als have the potential to further reduce rates of breast cancer in high-risk postmenopausal women. This article reviews the evidence to support the potential efficacy of Als in the chemoprevention setting. It particularly focuses on a discussion of novel concepts of utilising Als, so that they reduce breast cancer risk while minimising systemic toxicity, and highlights the importance of accurately developing risk prediction algorithms.
\end{abstract}

Endocrine-Related Cancer (2006) 13 827-837

\section{Introduction}

Breast cancer contributes to one-third of cancers in the female population of UK. Its incidence has continued to increase with latest data showing 41000 newly diagnosed cases and 13000 deaths per year (Cancer Research UK Cancer Stats Monograph 2004). Mortality has, in fact, fallen over the last 15 years, but in order to reduce both morbidity and mortality from breast cancer the ultimate aim would be primary prevention. Any intervention aimed at halting or slowing the natural history of early breast cancer must recognise and minimise the personal short- and long-term side effects of such actions. Although tamoxifen has remained the standard of care for adjuvant hormonal treatment for some time, recent data on the use of aromatase inhibitors (AIs) for early breast cancer in postmenopausal women has prompted interest in these drugs being used for chemoprevention.

\section{Chemoprevention of breast cancer - selective oestrogen receptor modulators (SERMs)}

Four trials have been presently reported on the use of tamoxifen (SERM) as a chemoprevention agent. The initial NSABP P-1 study published in 1998 found that the risk of invasive breast cancer was reduced by $49 \%$ $(P<0.00001)$ from 43.4 to 22.0 per 1000 women in those receiving tamoxifen compared with placebo (Fisher et al. 1998). This led to FDA approving the use of tamoxifen in chemoprevention for those at high risk scored by the Gail model. Updated outcome analysis in 2005 has not shown a great variation in these results (Fisher et al. 2005). In the most recent reported international chemoprevention study (IBIS-1), 7000 women with increased risk of breast cancer or pre-invasive cancer changes on biopsy were randomised to tamoxifen or placebo. In an overview analysis of tamoxifen prevention studies in 2003, outcome data from NSABP P-1 and IBIS-1 were considered along with the Royal Marsden Trial and the Italian study. Overall, there was a 38\% (95\% CI 28-46; $P<0.0001)$ reduction in the incidence of breast cancer for those who received tamoxifen compared with placebo (Cuzick et al. 2003). As anticipated, there was only a statistically significant difference in the incidence of ER-positive cancers (relative reduction 48 ; 95\% CI 36-58; $P<0.0001)$.

Raloxifene, another SERM, originally developed for the treatment of osteoporosis, has a different activity profile compared with tamoxifen. In the MORE study, two different doses of raloxifene were compared with placebo, in a study designed to assess bone protection with breast cancer as a secondary endpoint (Cummings et al. 1999). After a median follow-up of 40 months, there were fewer invasive cancers in those receiving 
raloxifene $(\mathrm{RR}=0.24,95 \% \mathrm{CI} 0.13-0.44 ; P<0.001)$ compared with placebo. The Continuing Outcomes Relevant to Evista (CORE) trial, which examined the effects of an additional 4 years of raloxifene (Evista) in those originally randomised to the active drug versus continued placebo, reported a similar magnitude of effect at 8 years with reduction of $66 \%$ in the raloxifene arm in invasive breast cancer $(\mathrm{HR}=0.34,95 \% \mathrm{CI}$ $0.22-0.50)$. There was no statistically significant reduction in ER-negative or non-invasive cancers (Martino et al. 2004).

The main limitation to the use of tamoxifen in a prevention-setting relates to its side-effect profile with thromboembolic events increased in all studies with relative risk of $1.9(1.4-2.6 ; P<0.0001)$ (Cuzick et al. 2003). In addition, increased rates of endometrial cancer were seen in all prevention studies with a consensus relative risk of $2.4(1.5-4.0 ; P=0.0005)$. Most excess risk is seen in cases with age 50 or older. Thus far, no increase in endometrial cancer but similar thromboembolic effects has been seen with raloxifene. Such toxicity data will be an important outcome measure in the ongoing STAR trial (tamoxifen versus raloxifene for 5 years in postmenopausal women), which will be reported at the American Society of Clinical Oncology Annual Meeting 2006.

\section{Uptake and compliance in prevention studies}

The attitude of women to chemoprevention is highly varied and has been examined in small focus groups and larger multicentre trials. In one cross-sectional survey of 355 high-risk women conducted in France, England and Canada, only 58\% expressed that they would consider embarking on a chemoprevention study (Julian-Reynier et al. 2001). Uncertainty of personal cancer risk and anxiety over treatment side effects are predictably among the highest perceived barriers to chemoprevention (Cyrus-David \& Strom 2001). High levels of compliance seen in chemoprevention studies (e.g. $80 \%$ in IBIS-1) are likely to be reduced in a general population setting where research support is not available. Along with the efficacy of any chemotherapeutic agent, researchers, health economists and public health bodies need to be aware of psychosocial barriers and compliance issues that will limit the overall effectiveness of large-scale chemoprevention projects.

\section{Als: mechanism of action}

AIs significantly suppress plasma oestrogen levels by inhibiting or inactivating aromatase, the enzyme, which catalyses the conversion of androgens to oestrogens. High levels of aromatase are found in the placenta and granulosa cells of ovarian follicles with lower levels in s.c. fat, liver, muscle, brain, normal and malignant breast tissue. Postmenopausal oestrogen production is from non-glandular sources, in particular, subcutaneous fat. Mean plasma levels of oestradiol fall from around $110 \mathrm{pg} / \mathrm{ml}(400 \mathrm{pmol} / \mathrm{l})$ to $7 \mathrm{pg} / \mathrm{ml}$ ( $25 \mathrm{pmol} / \mathrm{l})$ during menopause. Third-generation AIs are classified as type-1 (steroidal inactivator) e.g. letrozole, anastrozole; or type-2 (non-steroidal inhibitor) e.g. exemestane.

Measurement of oestrogen supression with AIs can be difficult with some assays due to the pre-existing low levels seen in postmenopausal women. Isotopic measurements of total body aromatisation have reported inhibition greater than $97 \%$ at clinical doses of all third generation AIs. There is evidence that letrozole causes more complete suppression of plasma oestrogen levels and inhibition of in vivo aromatisation than anastrozole (Geisler et al. 2002).

\section{Oestrogen carcinogenesis: implications for aromatase inhibition}

Oestrogen and its catechol metabolites are carcinogenic in various rodent tissues including mammary glands (Yue et al. 2003). Yager and Davidson have recently reviewed the potential role of oestrogen metabolites in human breast cancer in an extensive manner. Oxidative metabolism of oestrone and oestradiol by several cytochrome P450 enzymes has also been demonstrated in humans. The resulting oestrogen 3,4-quinone can form stable DNA adducts leading to mutations. Several studies have observed that oxidative metabolites of oestrogen have mutagenic and carcinogenic potential in cell line and xenograft models, however, this has not been definitively demonstrated in human breast cancer. Although oestrogen metabolites have been detected in human breast tissue, associated oxidative DNA damage, or oestrogen-quinone, adenine and guanine adducts have not been proven (Yager \& Davidson 2006).

Theoretically, therefore, AIs have a greater potential to prevent the development of de novo cancer by virtue of reducing oestrogen levels and in turn their damaging metabolites. This is expected to be a mechanism superior to tamoxifen, which inhibits oestrogen receptor-mediated function only. Data from the ATAC (anastrozole versus tamoxifen versus the combination) adjuvant study do not suggest this as being a major preventative mechanism for AIs as incidence of contralateral breast cancer with the 
combination arm was similar to tamoxifen and higher than anastrozole.

\section{Using Als for chemoprevention}

\section{Preclinical data}

There is evidence from xenograft models that AIs can be used to reduce the development of mammary carcinomas. Vorozole given over 42 days, reduced tumour growth by $90 \%$ in rats bearing dimethylbenzanthracene-induced oestrogen-dependent mammary tumours (De Coster et al. 1992). Similarly, vorozole and aminoglutethimide have proven effective in reducing incidence and multiplicity of $N$-methyl- $N$ nitrosourea (MNU)-induced mammary tumours in rats (Lubet et al. 1994, Moon et al. 1994).

\section{Adjuvant studies: reduction in contralateral breast cancer}

AIs have shown superiority to tamoxifen in the neoadjuvant and metastatic settings and are the firstline endocrine therapy of choice for these ER-positive patients (Smith \& Dowsett 2003). Superiority has also been shown in adjuvant trials and their inclusion in the adjuvant treatment of postmenopausal ER-positive patients is recommended (Winer et al. 2003). Adjuvant studies with AIs have shown a greater reduction in contralateral breast cancers than tamoxifen to further encourage their role in the prevention setting (Fig. 1). In the ATAC trial (anastrozole alone or in combination with tamoxifen versus tamoxifen for postmenopausal women with early breast cancer), there was a $58 \%$ reduction in the odds of developing primary contralateral breast cancer in the anastrozole group compared with tamoxifen (Howell 2005). In the first two arms of the BIG-98 study, to report (letrozole versus tamoxifen), contralateral breast primaries were seen in $0.4 \%$ of those on letrozole compared with $0.7 \%$ on tamoxifen (Thürlimann 2005). Data from the Intergroup Exemestane Study (IES) of exemestane following 2-3 years of tamoxifen in postmenopausal women support these findings with a statistically significant reduction in contralateral breast cancer in those receiving the AI versus tamoxifen (9 vs 20 respectively, $P=0.004$; Coombes et al. 2004). In cases where letrozole was compared to placebo, after 5 years of tamoxifen, in early breast cancer (MA-17), there was a similar reduction of $39 \%$ in the incidence of developing contralateral disease in favour of letrozole (Goss et al. 2005).

\section{Side effects of Als}

Overall, AIs have a favourable toxicity profile compared with tamoxifen. In the ATAC trial (which has the most mature follow-up data), the use of anastrozole was

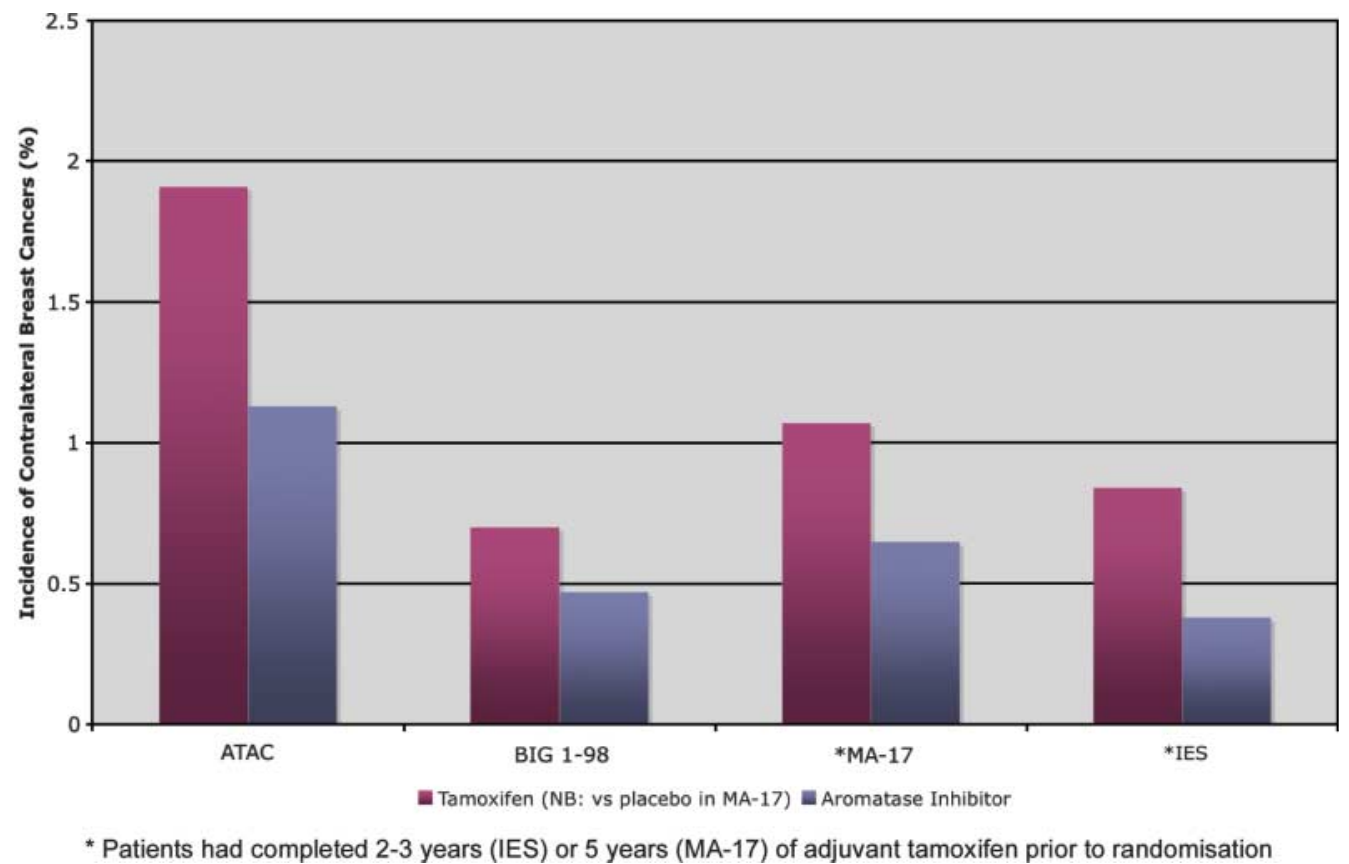

Figure 1 Incidence of contralateral breast cancers in adjuvant aromatase inhibitor trials. 
associated with a reduction in incidence of endometrial cancer compared to tamoxifen ( 0.2 vs $0.8 \%$ OR 0.29 ; $P=0.02$ ) (Howell 2005). Vaginal bleeding is also decreased with AIs in other studies but rates of endometrial cancer are not reported. In the ATAC, BIG1-98 and IES studies, thromboembolic events were statistically significantly lower in all those receiving AIs in comparison with tamoxifen.

Adjuvant studies have shown a higher level of skeletal morbidity secondary to AIs compared with tamoxifen presumably related to long-term oestrogen suppression. The ATAC study reported a fracture rate of 5.9 vs $3.7 \%$ for anastrozole versus tamoxifen alone respectively $(P<0.0001)$. The recently published adjuvant study of exemestane versus tamoxifen after 3 years of tamoxifen in postmenopausal women also showed a trend toward a greater level of osteoporosis in the exemestane arm (7.4 vs 5.7\%; $P=0.05$ ). Tamoxifen is, however, known to have bone-protection effects, therefore, the proportion of the higher level that would be seen in fracture rate, if compared to a normal untreated population, is unclear. In one randomised trial of AI versus placebo in a low-risk group, those on exemestane did not experience a deleterious effect on bone mineral density (BMD) compared with placebo. However, there was a higher than anticipated loss of BMD in the placebo group (Lonning et al. 2004). In the MA-17 trial, there was a trend toward increase in fractures (3.6 vs $2.9 \%$; $P=0.24)$ and osteoporosis (5.8 vs $4.5 \% ; P=0.07)$ in the letrozole arm. This study was, however, terminated early at a median follow-up of 2.4 years due to the positive impact on disease-free survival of letrozole; therefore, long-term safety data will not be available. It is anticipated that all healthy women using AIs for chemoprevention will obligatorily have bone densiometry assessment and if merited, concomitant bisphosphonates.

As oestrogen is known to reduce atherogenic total cholesterol and LDL-cholesterol whilst favourably increasing HDL-cholesterol, there has been concern that oestrogen suppression associated with AIs would have an adverse effect on lipid levels. Substudies within adjuvant AI trials have attempted to address this issue. The MA-17 study provides a good vehicle for assessing these effects, as it is placebo controlled, although previous tamoxifen therapy may exert some influence. Lipid levels were assessed at baseline, 6 and 12 months and annually thereafter. In this group of 347 nonhyperlipidaemic women, there were marginal changes in HDL-cholesterol at 6 months $(P=0.049)$, LDL cholesterol at 12 months $(P=0.033)$ and triglycerides at 24 months $(P=0.036)$. However, there was no statistically significant difference in the number of patients exceeding the lipid parameter thresholds (Wasan et al. 2005). The neoadjuvant IMPACT study (run-in sequence with ATAC) randomised women to anastrozole, tamoxifen or a combination of both. Nonfasting blood levels assessed at baseline, 2 and 12 weeks did not demonstrate a detrimental effect of anastrozole, with a significant rise in HDL-cholesterol $(11.2 \%)$, a non-significant rise in total cholesterol $(6.5 \%)$ and nonHDL-cholesterol (3.4\%) (Banerjee et al. 2005). There was a trend toward reduction of HDL with exemestane, when measured over a 12-month period of the TEAM (Tamoxifen and Exemestane Adjuvant Multicentre) trial. However, the atherogenic risk determined by the total cholesterol:HDL ratio remained stable in both the arms throughout treatment (Markopoulos et al. 2005). Although these subprotocols compare different AIs with different comparative arms, it appears that the initial concerns over their impact on lipid profiles may be unfounded. Preliminary data from the randomised multicentre LEAP (letrozole, exemestane and anastrozole pharmacodynamics) study in healthy postmenopausal women found a detrimental impact on 'pro-atherogenic LDL:HDL ratio' associated with exemestane ( $P=0.047$ at 3 months), a trend towards increased triglycerides with letrozole but no harmful lipid changes associated with anastrozole McCloskey et al. 2005). It remains unclear whether these effects are reversible or ultimately would lead to an increase in cardiovascular disease.

In addition to objective clinically quantifiable endpoints, patient-scored quality of life (QoL) data have been obtained from subprotocols within the ATAC, IES and MA-17 studies. Direct comparisons between these trials are, however, limited by the different time-scales, patient populations and comparative arm examined. In none of the studies was there a significant difference between QoL scores in the AI and the comparative arm. The well-validated Functional Assessment of Cancer Therapy-Breast (FACT-B) and endocrine subscale (ES) (Brady et al. 1997) were used in the ATAC and IES substudies. In the ATAC study, endocrine QoL scores were seen to deteriorate over 3 months, gradually improve and plateau by 2 years (Fallowfield 2005). MA-17 and A-TAC reported a worsening of vasomotor symptoms, vaginal dryness and sexual dysfunction in the AI arm compared with tamoxifen or placebo. This was not a statistically significant finding in the exemestane arm of IES (Fallowfield et al. 2006). There are often discrepancies between physician and patient-scored QoL scales. Analysis of validated QoL measures is an 
essential component of determining optimum care for women, particularly in the chemoprevention setting.

\section{Ongoing Al prevention studies}

The IBIS-2 randomised phase III trial compares anastrozole versus placebo over 5 years in 6000 women at high risk. A further 4000 women, who have been previously treated for DCIS, are randomised to receive tamoxifen or anastrozole. In addition to incidence of breast cancer as a primary endpoint, this trial is also investigating disease-specific mortality along with bone mineral density and other end-organ functions. Women deemed osteopenic at the beginning of the study will be randomised to observation or continued bisphosphonate therapy. Following the adjuvant IES exemestane study and incorporating theoretical data on the use of COX-2selective inhibitors in chemoprevention, the NCIC CTG MAP-3 study was launched. This randomised women to exemestane with or without celecoxib versus placebo. Unfortunately, due to recent data on increased cardiac death associated with COX-2 selective inhibitors, the celecoxib arm has closed. In Italy, exemestane is also being evaluated compared to placebo over 3 years in postmenopausal women with know BRCA $1 / 2$ gene mutations.

\section{Novel concepts for Al chemoprevention}

On balance, the superior efficacy and tolerability of AIs make them a better potential chemopreventive agent than tamoxifen in postmenopausal high-risk women. However, as with all drugs, they are not devoid of side effects and consideration of this has encouraged the development of novel ideas for optimising their use as outlined below.

\section{Systemic versus breast aromatase activity}

In addition to endogenous and exogenous systemic oestrogen, breast tissue aromatase activity has been implicated in the pathogenesis of hormone-dependent breast cancer. The biology and clinical implications of intratumoral aromatase and oestrogens have been reviewed by Geisler (2003) and Thijssen (2004). The concentration of oestradiol in breast carcinoma tissue in postmenopausal women has been shown to be approximately ten times that in plasma; some of this gradient is attributable to uptake but much of it to in situ synthesis, the enzymes required for conversion of androgens to oestradiol including aromatase, are present in most breast carcinomas. Such autocrine production of oestradiol is highly variable with up to a 40 -fold range between highest and lowest levels in mammary adipose tissue and breast cancers. Most, but not all, studies indicate that aromatase activity is higher in the quadrant of the breast where the tumour is localised than in other quadrants, although it is unclear whether this might be causative of increased breast cancer incidence in that quadrant or due to the presence of the tumour in that quadrant, e.g. by release of factors from the tumour-stimulating aromatase activity (James et al. 1987, Thijssen et al. 1991). Tumoural aromatase status has been shown to correlate with response to the AI aminoglutethimide; however, this is a small study from which definitive conclusions cannot be made. (Miller \& O'Neill 1987). The in vivo quantitative importance of intratumoural aromatase has been elegantly assessed by Miller and colleagues using infusion of different labels on androstenedione and oestrone prior to biopsy of breast cancer (Miller et al. 2002). This revealed that almost all tumours gained oestrogen via both local and peripheral aromatase. While the average was about 50:50 from both sources, the range was from 0 to $100 \%$. Use of modern AIs clearly suppresses intratumoral oestrogen levels markedly (Geisler et al. 2001). However, it appears that aromatase production in breast cancer tissue may be under the control of different transcriptional promoters than normal tissue (Simpson \& Dowsett 2002). There is therefore the potential to exploit differential oestradiol production between breast and systemic tissues when exploring AIs or aromatase suppressants in the chemoprevention setting as discussed below.

\section{Partial oestradiol suppression}

Reduction rather than elimination of oestradiol is an attractive concept for chemoprevention, since this provides the possibility that a risk reduction benefit could be achieved without bone and other toxicities. This might be achieved in one of two ways. First, the log-linear response curve seen between AI dose and oestradiol suppression, means that considerable oestradiol suppression can be achieved at doses much lower than those conventionally used for treatment. A single dose of $0.1 \mathrm{mg}$ letrozole can suppress oestradiol levels by $77 \%$ for up to 3 days in postmenopausal women (Iveson et al. 1993). Comparison of three doses of the non-steroidal inhibitor fadrozole (CGS 16949A) found significant suppression of oestradiol at 0.3, 1.0 and $2.0 \mathrm{mg}$ twice everyday. Even at the lowest dose, mean suppression of oestradiol at day 14 was 54\% (Dowsett et al. 1990). Such studies have demonstrated marked intersubject heterogeneity, suggesting that use of low-dose AI might require individual titration with consequent feasibility issues. 
Secondly, there is the potential to suppress endogenous oestrogens both peripherally and within the breast by the action of AIs then deliver exogenous oestrogens at low doses to give a physiological dose to most tissues with continued reduction of oestrogen within the breast. Oral hormone-replacement therapy (HRT) with oestradiol leads to systemic availability of only $2-10 \%$ oestradiol due to gut and liver metabolism. Transdermal patches avoiding first-pass hepatic metabolism give a systemic availability of oestradiol in the order of $>90 \%$. Standard manufactured 'low-dose patches' are generally twice a week preparations of 25 or $50 \mathrm{mcg}$ (delivering serum levels of approximately 60-120 pmol/l). Menostar, a $14 \mathrm{mcg}$ weekly patch, has been specifically developed for prophylaxis of osteoporosis rather than alleviation of systemic climacteric symptoms. In a placebo-controlled trial $(n=417)$, the Menostar group had statistically significant improvements in lumbar spine and hip bone density with an oestradiol rise from a median of $17.6 \mathrm{pmol} / \mathrm{l}$ at baseline to $31 \mathrm{pmol} / \mathrm{l}$ at 2 years $(P<0.001)$ (Ettinger et al. 2004). We are presently investigating the reproducibility of systemic oestradiol levels in postmenopausal women receiving an $\mathrm{AI}$ in combination with a lowdose oestradiol patch. Secondary endpoints of bone resorption marker CTX and lipid profiles will also be assessed. Ultimately, this approach needs to be validated by measuring breast tissue oestrogen levels pre- and post-AI therapy, and before and after giving systemic oestrogen. However, there are practical and ethical limitations to successfully performing core-cut biopsies from normal breast tissue.

\section{Targeted oestradiol supression}

Attempts have been made to develop transdermal AIs. With steroidal aromatase inactivators it is possible that the drug would localise preferentially in the breast to reduce oestrogen production locally, with markedly reduced systemic effects. There are, however, no published data on this approach.

\section{Aromatase inhibition in premenopausal women}

AIs are not used in the treatment of premenopausal women with breast cancer (except in combination with GnRH agonists) as oestradiol levels are not consistently suppressed to a postmenopausal level, and preclinical studies have shown that the associated gonadal stimulation can lead to the development of multiple ovarian follicles. However, in the context of chemoprevention, it is possible that this could be turned to an advantage with a low-dose AI resulting in the suppression of oestradiol in breast tissue, and systemic effects being compensated for by stimulation of gonadal synthesis of oestrogens.

The effect of aromatase inhibition on breast tumour proliferation and systemic hormone levels has been investigated in a pre-surgical 2-week study in 30 premenopausal women (Dowsett \& Haynes 2003). The third-generation AI, YM511, caused highly variable effects with some suppression of oestradiol to less than premenopausal levels in some of the women and marked stimulation to supra-normal levels in others. There was a greater effect on plasma oestrone levels than oestradiol consistent with oestradiol being a more predominant ovarian oestrogen subject to feedback regulation. In this small number of breast cancers assessed, Ki-67 was not significantly reduced, which is in contrast to the $>80 \%$ suppression seen in postmenopausal women in a similar setting (Harper-Wynne et al. 2002a). Thus, intratumoural aromatase inhibition was not sufficient to result in a marked anti-proliferative effect and the variability between women means this approach is unlikely to be successful in the prevention setting.

\section{Cyclooxgenase-2 (COX-2)selective inhibitors}

There have been several epidemiological and observational studies that have reported an inverse relationship between colorectal cancers and adenomas with the use of non-steroidal anti-inflammatory drugs (NSAIDS). Although less compelling, there are both prospective and case-control studies, which would suggest a similar beneficial effect in breast cancer (Davies et al. 2002). The prominent NSAID target is cyclooxygenase, which exists in two main isoforms: the constitutive COX-1 and COX-2, which is induced by a variety of stimuli including tumour promoters, endotoxin, cytokines and hormones. NSAIDs have also been demonstrated to have COX-independent activity that may be implicated in their anti-cancer effect $(\mathrm{He}$ et al. 1999).

COX-2 expression has been linked with the features of poor prognosis breast cancers including large tumour size, the presence of axillary lymph node metastases and HER-2 status (Arun \& Goss 2004). Its expression has also been correlated with aromatase expression in human breast cancers. This association may in part explain mammary carcinogenesis by induction of aromatase by prostaglandin E2 (Brueggemeier et al. 1999). COX-2 expression is also associated with angiogenic and anti-apoptotic properties in tumours such that their role in chemoprevention is potentially via these routes as well as inhibition of oestrogen synthesis (Hsu et al. 2000, Masferrer et al. 


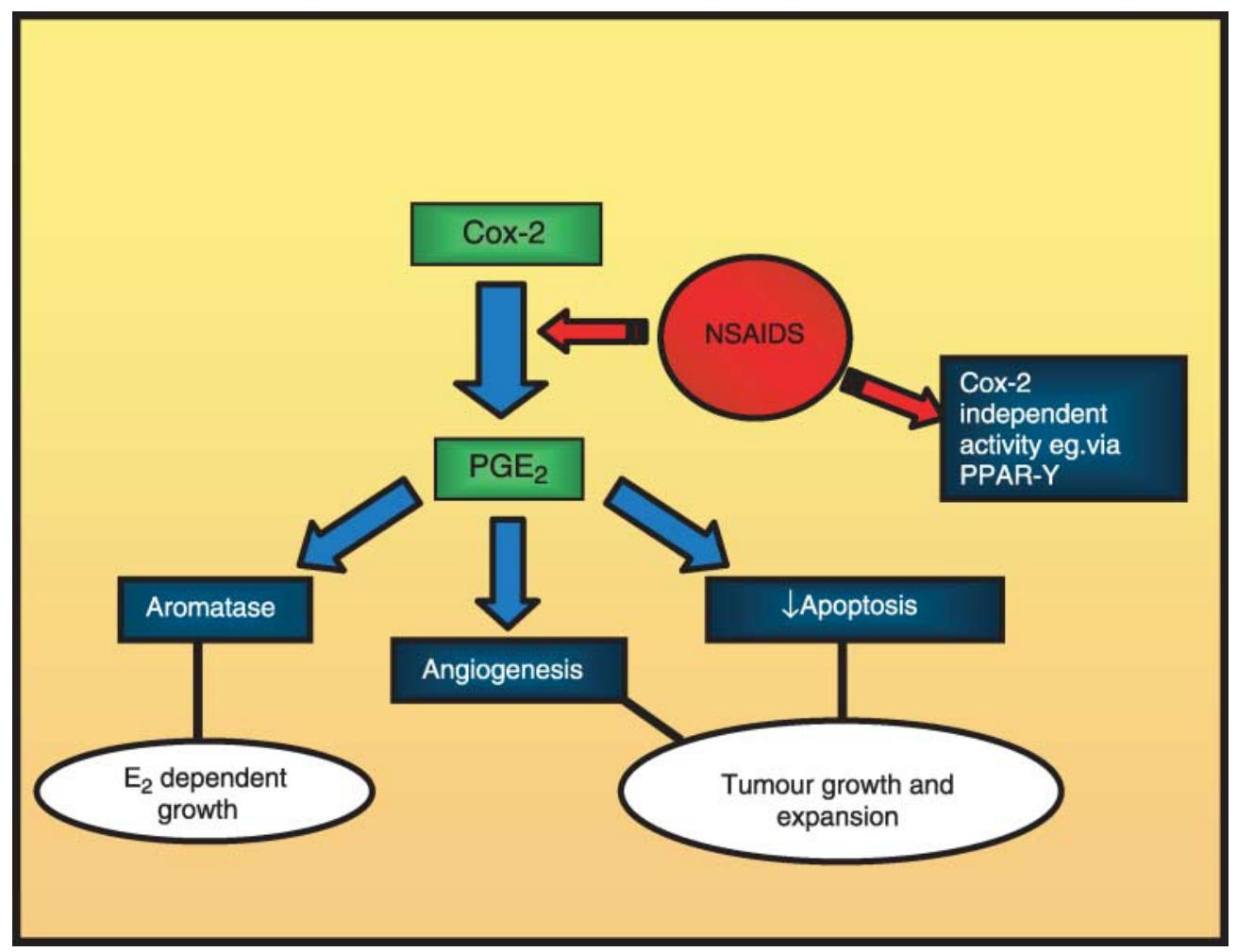

Figure 2 Potential role of Non-Steroidal Anti-inflammatory drugs (NSAIDs) in Breast Cancer Chemoprevention.

2000). However, as alluded earlier, unless cardiotoxicity issues related to these drugs are resolved, their place in chemoprevention in a healthy population is clearly in doubt (Fig. 2).

\section{Prevention surrogate biomarkers and targeting strategies Biomarkers for risk prediction}

There is great interest in defining biomarkers that can be incorporated both into breast cancer risk models and also evaluable as markers of response to prevention strategies. Ideally, such biomarkers must be present in a reasonable proportion of at-risk individuals, be easily obtainable, biologically and statistically associated with a reversible phase of carcinogenesis and seen to change with known effective chemopreventive agents. Various surrogate end-point biomarkers have been proposed, such as mammographic density, serum-sex hormone levels and serum insulin-like growth factor.

Breast tissue biomarkers are thought to most directly reflect the susceptibility of the breast to the neoplastic process, however, obtaining these is impaired by numerous technical difficulties. Fabian and colleagues have pioneered the approach of random periareolar fine needle aspiration cytology. They have demonstrated that presence of hyperplasia with atypia along with 10-year Gail risk can be used to predict a cohort of women with very high short-term risk (Fabian et al. 2000). Harper-Wynne et al. (2002b) used targeted core-cut biopsies of normal breast tissue pre- and post3 months of letrozole to define changes in the proliferation marker Ki-67. Although there was a decrease in median Ki-67 count from 1.28 to $0.99 \%$, this did not reach statistical significance $(P=0.65)$. This study confirmed the significant inherent complications in using normal breast tissue from postmenopausal women where paucity of epithelial cells impedes the utility of conventional immunohistochemical biomarkers.

\section{Potential target populations for risk prevention strategies}

Strategies are needed to better identify women at risk of hormone-sensitive breast cancers. By doing so, AI intervention is more likely to give a favourable cancer reduction to side-effect ratio. The multifactorial Gail model is the most widely used in clinical trials. It utilises several reproductive variables along with family history and any known breast biopsy result, 
and was originally designed to estimate risk in populations. It performed well in predicting incidence of breast cancers in the Nurses Health Study cohort and the Breast Cancer Prevention Trial (BCPT) (Spiegelman et al. 1994). However, it is not as robust in determining individual risk, and as such, its use is limited in direct patient consultations and predicting numbers needed to treat. Women with atypical ductal hyperplasia, atypical lobular hyperplasia or lobular carcinoma in situ given tamoxifen in the NSABP-P1 prevention study had $>49 \%$ reduction in breast cancer incidence. These lesions are particularly associated with oestrogen receptor-positive breast cancers and as such women with these diagnoses, who are often highly motivated to participate in risk prevention studies. Mammographic breast density has repeatedly been shown to be an independent risk factor for breast cancer (Boyd et al. 2005). More recently, data have shown that this can be modulated by tamoxifen use and as such may be an important eligibility criteria and endpoint in prevention studies (Cuzick et al. 2004).

Several groups have published prospective data on endogenous hormones and breast cancer but none have individually been extensive enough to give accurate estimates of risk. The Endogenous Hormones and Breast Cancer Collaborative Group (Key et al. 2002) was set up to analyse the original pooled data from nine such trials. The potential influence of other variables on the association between hormone concentration and breast cancer risk was examined by adjusting for established risk factors, including age at menarche, parity, previous use of oral contraceptives, type of menopause and BMI. The relative risk of breast cancer for women, whose oestradiol levels were in the top quintile compared with those in the bottom quintile, was 2.00 (95\% CI, 1.47-2.71). Within the MORE study, reduction in the risk of breast cancer was more notable in women with the highest quartile of oestradiol levels (Cummings et al. 2002). Although this adds further weight to the pooled analysis, the relationship in high-risk groups is not clear. Using a case-cohort design, a subgroup of women in the NSABP-P1 trial, who received placebo were not shown to have an increased relative risk of breast cancer associated with sex hormone levels. There was a high level of atypical ductal hyperplasia in women in this study, which when combined with other risk factors may outweigh any effect of endogenous sex hormones. In addition, other confounding factors e.g. baseline hormone levels and variation between breast and systemic oestrogen levels may be significant factors in this group (Beattie et al. 2006). Interestingly, a prospective case-control study within the Nurses Health Study classifying women into low- or high-risk based on family history, Gail, Rosner and Colditz criteria found that oestradiol appeared more strongly associated with breast cancer in the higher predicted risk group $(\mathrm{RR}=4.5 ; 95 \% \mathrm{CI}, 2.1-9.5)$ than those in the lower risk group $(\mathrm{RR}=2.1 ; 95 \% \mathrm{CI} 1.2-3.6)$ (Eliassen et al. 2006). Endogenous sex hormone levels should be considered a valuable tool in the construction of risk algorithms but prospective evaluation of their impact in high-risk groups needs further evaluation (Fig. 3).

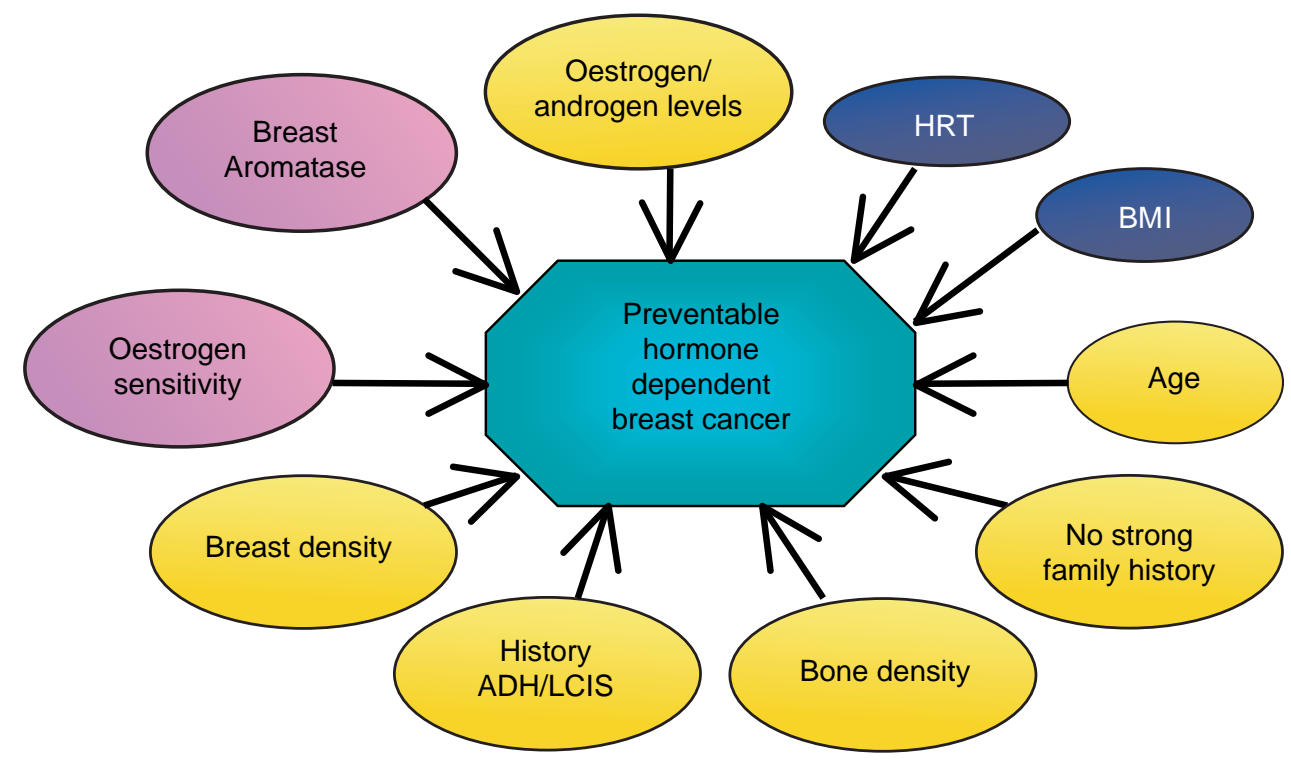

Figure 3 Possible components to include in hormone-dependent breast cancer risk algorithm. 


\section{Conclusions}

Given the greater reduction in contralateral breast cancer with AIs than tamoxifen in adjuvant trials, it is probable they will perform well in on-going chemoprevention trials. The most significant issue will be the benefit, side effect ratio in a healthy population. There are novel approaches to AI usage that may improve this ratio. Defining, who will benefit most, and how to utilise AIs best will assist in identifying their role. Those healthy women, who are likely to gain most from such an intervention may be the same group, who have the most to lose in quality of life scores and longterm morbidity.

\section{Acknowledgements}

The authors declare that there is no conflict of interest that would prejudice the impartiality of this scientific work.

\section{References}

Arun B \& Goss P 2004 The role of COX-2 inhibition in breast cancer treatment and prevention. Seminars in Oncology $3122-27$.

Banerjee S, Smith IE, Folkerd L, Iqbal J, Barker P \& Dowsett IMPACT trialists M 2005 Comparative effects of anastrozole, tamoxifen alone and in combination on plasma lipids and bone-derived resorption during neoadjuvant therapy in the impact trial. Annals of Oncology 16 1632-1638.

Beattie MS, Costantino JP, Cummings SR, Wickerham DL, Vogel VG, Dowsett M, Folkerd EJ, Willett WC, Wolmark N \& Hankinson SE 2006 Endogenous sex hormones, breast cancer risk, and tamoxifen response: an ancillary study in the NSABP breast cancer prevention trial (P-1). Journal of the National Cancer Institute 98 110-115.

Boyd NF, Rommens JM, Vogt K, Lee V, Hopper JL, Yaffe MJ \& Paterson AD 2005 Mammographic breast density as an intermediate phenotype for breast cancer. The Lancet Oncology 6 798-808.

Brady MJ, Cella DF, Mo F, Bonomi AE, Tulsky DS, Lloyd SR, Deasy S, Cobleigh M \& Shiomoto G 1997 Reliability and validity of the functional assessment of cancer therapy-breast quality-of-life instrument. Journal of Clinical Oncology 15 974-986.

Brueggemeier RW, Quinn AL, Parrett ML, Joarder FS, Harris RE \& Robertson FM 1999 Correlation of aromatase and cyclooxygenase gene expression in human breast cancer specimens. Cancer Letters 140 27-35.

Cancer Research UK 2004 UK mortality and incidence of breast cancer. Cancer Stats Monograph 21. ISBN 0-9546256-2-5.

Coombes for the Intergroup Exemestane Study RC 2004 A randomized trial of exemestane after 2 to 3 years of tamoxifen therapy in postmenopausal women with primary breast cancer. New England Journal of Medicine 350 1081-1092.

Cummings SR, Eckert S, Krueger KA, Grady D, Powles TJ, Cauley JA, Norton L, Nickelsen T, Bjarnason NH, Morrow M et al. 1999 The effect of raloxifene on risk of breast cancer in post-menopausal women. Results from the MORE randomised trial. JAMA 281 2189-2197.

Cummings SR, Duong T, Kenyon E, Cauley JA, Whitehead M \& Krueger KA 2002 Multiple outcomes of raloxifene evaluation (MORE) Trial Serum estradiol level and risk of breast cancer during treatment with raloxifene. JAMA 287 216-220.

Cuzick J, Powles T, Veronesi U, Forbes J, Edwards R, Ashley S \& Boyle P 2003 Overview of the main outcomes in breast cancer prevention trials. The Lancet 361 296-300.

Cuzick J, Warwick J, Pinney E, Warren RM \& Duffy SW 2004 Tamoxifen and breast density in women at increased risk of breast cancer. Journal of the National Cancer Institute 96 2004. 621-8JNCI.

Cyrus-David MS \& Strom SS 2001 Chemoprevention of breast cancer with selective estrogen receptor modulators: views from broadly diverse focus groups of women with elevated risk for breast cancer. Psychooncology 10 521-533.

De Coster R, Van Ginckel RF, Callens MJ, Goeminne NK \& Janssens BL 1992 Antitumoral and endocrine effects of $(+)$-vorozole in rats bearing dimethylbenzanthracene-induced mammary tumors. Cancer Research 52 1240-1244.

Davies G, Martin LA, Sacks N \& Dowsett M 2002 Cyclooxygenase-2 (COX-2), aromatase and breast cancer: a possible role for COX-2 inhibitors in breast cancer chemoprevention. Annals of Oncology 13 669-678.

Dowsett M, Stein RC, Mehta A \& Coombes RC 1990 Potency and selectivity of the non-steroidal aromatase inhibitor CGS 16949A in postmenopausal breast cancer patients. Clinical Endocrinology 32 623-634.

Dowsett M \& Haynes BP 2003 Hormonal effects of aromatase inhibitors: focus on premenopausal effects and interaction with tamoxifen. Journal of Steroid Biochemistry and Molecular Biology 86 255-263.

Eliassen AH, Missmer SA, Tworoger SS \& Hankinson SE 2006 Endogenous steroid hormone concentrations and risk of breast cancer: does the association vary by a woman's predicted breast cancer risk? Journal of Clinical Oncology 24 1823-1830.

Ettinger B, Ensrud KE, Wallace R, Johnson KC, Cummings SR, Yankov V, Vittinghoff E \& Grady D 2004 Effects of ultralow-dose transdermal estradiol on bone mineral density: a randomized clinical trial. Obstetrics and Gynecology 104 1823-1830.

Fabian CJ, Kimler BF, Zalles CM, Klemp JR, Kamel S, Zeiger S \& Mayo MS 2000 Short term breast cancer prediction by random periareolar fine needle aspiration cytology and the Gail risk model. Journal of the National Cancer Institute 92 1217-1227. 
Fallowfield L 2005 Acceptance of adjuvant therapy and quality of life issues. The Breast 14 612-616.

Fallowfield LJ, Bliss JM, Porter LS, Price MH, Snowdon CF, Jones SE, Coombes RC \& Hall E 2006 Quality of life in the intergroup exemestane study: a randomized trial of exemestane versus continued tamoxifen after 2-3 years of tamoxifen in postmenopausal women with primary breast cancer. Journal of Clinical Oncology 24 910-917.

Fisher B, Costantino JP, Wickerham DL, Redmond CK, Kavanah M, Cronin WM, Vogel V, Robidoux A, Dimitrov N, Atkins J et al. 1998 Tamoxifen for prevention of breast cancer: report of the National Surgical Adjuvant Breast and bowel project P-1 study. Journal of the National Cancer Institute 90 1371-1388.

Fisher B, Costantino JP, Wickerham DL, Cecchini RS, Cronin WM, Robidoux A, Bevers TB, Kavanah MT, Atkins JN, Margolese RG et al. 2005 Tamoxifen for the prevention of breast cancer: current status of the National Surgical Adjuvant Breast and Bowel Project P-1 study. Journal of the National Cancer Institute 97 1636-1637.

Geisler J 2003 Breast cancer tissue estrogens and their manipulation with aromatase inhibitors and inactivators. Journal of Steroid Biochemistry and Molecular Biology 86 245-253.

Geisler J, Detre S, Berntsen H, Ottestad L, Lindtjorn B, Dowsett M \& Einstein Lonning P 2001 Influence of neoadjuvant anastrozole (Arimidex) on intratumoral estrogen levels and proliferation markers in patients with locally advanced breast cancer. Clinical Cancer Research 7 1230-1236.

Geisler J, Haynes B, Anker G, Dowsett M \& Lonning PE 2002 Influence on letrozole and Anastrozole on total body aromatization and plasma estrogen levels in postmenopausal breast cancer patients evaluated in a randomized, cross-over study. Journal d'Odontologie Conservatrice 20 751-757.

Goss PE, Ingle JN, Martino S, Robert NJ, Muss HB, Piccart MJ, Castiglione M, Tu D, Shepherd LE, Pritchard KI et al. 2005 Randomized trial of letrozole following tamoxifen as extended adjuvant therapy in receptor-positive breast cancer: updated findings from NCIC CTG MA.17. Journal of the National Cancer Institute 97 1262-1271.

Harper-Wynne CL, Sacks NP, Shenton K, MacNeill FA, Sauven P, Laidlaw IJ, Rayter Z, Miall S, Howes A, Salter J et al. 2002a Comparison of the systemic and intratumoral effects of tamoxifen and the aromatase inhibitor vorozole in postmenopausal patients with primary breast cancer. Journal of Clinical Oncology 20 1026-1035.

Harper-Wynne C, Ross G, Sacks N, Salter J, Nasiri N, Iqbal J, A'Hern R \& Dowsett M 2002b Effects of the aromatase inhibitor letrozole on normal breast epithelial cell proliferation and metabolic indices in postmenopausal women: a pilot study for breast cancer prevention. Cancer Epidemiology, Biomarkers and Prevention 11 614-621.
He TC, Chan TA, Vogelstein B \& Kinzler KW 1999 PPAR- $\gamma$ is an APC regulated target of nonsteroidal anti-inflammatory drugs. Cell 99 335-345.

Hsu AL, Ching TT, Wang DS, Song X, Rangnekar VM \& Chen CS 2000 The cyclooxygenase-2 inhibitor celecoxib induces apoptosis by blocking Akt activation in human prostate cancer cells independently of Bcl-2. Journal of Biological Chemistry 275 11397-11403.

Howell A 2005 ATAC trialists group results of the ATAC (Arimidex, Tamoxifen, Alone or in Combination) trial after completion of 5 years' adjuvant treatment for breast cancer. The Lancet 365 60-63.

Iveson TJ, Smith IE, Ahern J, Smithers DA, Trunet PF \& Dowsett M 1993 Phase I study of the oral nonsteroidal aromatase inhibitor CGS 20267 in healthy postmenopausal women. Journal of Clinical Endocrinology and Metabolism 77 316-318.

James VH, McNeill JM, Lai LC, Newton CJ, Ghilchik MW \& Reed MJ 1987 Aromatase activity in normal breast and breast tumor tissues: in vivo and in vitro studies. Steroids 50 269-279.

Julian-Reynier CM, Bouchard LJ, Evans DG, Eisinger FA, Foulkes WD, Kerr B, Blancquaert IR, Moatti JP \& Sobol HH 2001 Women's attitudes toward preventive strategies for hereditary breast or ovarian carcinoma differ from one country to another: differences among English, French, and Canadian women. Cancer 92 959-968.

Key T, Appleby P, Barnes I \& Reeves G 2002 Endogenous hormones and breast cancer collaborative group endogenous sex hormones and breast cancer in postmenopausal women: reanalysis of nine prospective studies. Journal of the National Cancer Institute 94 606-616.

Lonning PE, Geisler J, Krag L, Ottestad L, Bremnes Y, Hagen AI, Schlichting E, Polli A, Paolini J \& Massimini G 2004 Effect of exemestane on bone: randomised placebo controlled study in postmenopausal women with early breast cancer at low risk. Proceedings of the American Society of Clinical Oncology 1636-1637. Abstract 518.

Lubet RA, Steele VE, Casebolt TL, Eto I, Kelloff GJ \& Grubbs CJ 1994 Chemopreventive effects of the aromatase inhibitors vorozole (R-83842) and 4-hydroxyandrostenedione in the methylnitrosourea (MNU)-induced mammary tumor model in SpragueDawley rats. Carcinogenesis 15 2775-2780.

Markopoulos C, Polychronis A, Zobolas V, Xepapadakis G, Papadiamantis J, Koukouras D, Lappas H \& Gogas H 2005 The effect of exemestane on the lipidemic profile of postmenopausal early breast cancer patients: preliminary results of the TEAM Greek sub-study. Breast Cancer Research and Treatment 93 61-66.

Martino S, Cauley JA, Barrett-Connor E, Powles TJ, Mershon J, Disch D, Secrest RJ \& Cummings SR 2004 Continuing outcomes relevant to Evista: breast cancer incidence in postmenopausal osteoporotic women in a randomized trial of raloxifene. Journal of the National Cancer Institute 96 1751-1761. 
Masferrer JL, Leahy KM, Koki AT, Zweifel BS, Settle SL, Woerner BM, Edwards DA, Flickinger AG, Moore RJ \& Seibert K 2000 Antiangiogenic and antitumor activities of cyclooxygenase-2 inhibitors. Cancer Research 60 1306-1311.

McCloskey E, Eastell R, Lakner G, Miyamoto A \& Clack G 2005 Initial results from the LEAP study: the first direct comparison of safety parameters between aromatase inhibitors in healthy postmenopausal women. San Antonio Breast Cancer Symposium. Abstract 2052.

Miller WR \& O'Neill J 1987 The importance of local synthesis of estrogen within the breast. Steroids 50 537-548.

Miller WR, Stuart M, Sahmoud T \& Dixon JM 2002 Anastrozole ('Arimidex') blocks oestrogen synthesis both peripherally and within the breast in postmenopausal women with large operable breast cancer. British Journal of Cancer 87 950-955.

Moon RC, Steele VE, Kelloff GJ, Thomas CF, Detrisac CJ, Mehta RG \& Lubet RA 1994 Chemoprevention of MNU-induced mammary tumorigenesis by hormone response modifiers: toremifene, RU 16117, tamoxifen, aminoglutethimide and progesterone. Anticancer Research 14 889-893.

Simpson ER \& Dowsett M 2002 Aromatase and its inhibitors: significance for breast cancer therapy. Recent Progress in Hormone Research 57 317-338.

Smith IE \& Dowsett M 2003 Aromatase inhibitors in breast cancer. NEJM 24 2431-2442.

Spiegelman D, Colditz GA, Hunter D \& Hertzmark E 1994 Validation of the Gail et al. model for predicting individual breast cancer risk. Journal of the National Cancer Institute 86 600-607.

Thijssen JH 2004 Local biosynthesis and metabolism of oestrogens in the human breast. Maturitas 49 25-33.
Thijssen JH, Blankenstein MA, Donker GH \& Daroszewski J 1991 Endogenous steroid hormones and local aromatase activity in the breast. Journal of Steroid Biochemistry and Molecular Biology 39 799-804.

Thürlimann B, on behalf of The Writing Committee B 2005 The breast international group (BIG 1-98) collaborative group. A comparison of letrozole and tamoxifen in postmenopausal women with early breast cancer. New England Journal of Medicine 26 2747-2757.

Wasan KM, Goss PE, Pritchard PH, Shepherd L, Palmer MJ, Liu S, Tu D, Ingle JN, Heath M, Deangelis D et al. 2005 The influence of letrozole on serum lipid concentrations in postmenopausal women with primary breast cancer who have completed 5 years of adjuvant tamoxifen (NCIC CTG MA.17L). Annals of Oncology 16 707-715.

Winer EP, Hudis C, Burstein HJ, Bryant J, Chlebowski RT, Ingle JN, Edge SB, Mamounas EP, Gelber R, Gralow J et al. 2003 American Society of Clinical Oncology technology assessment working group update: use of aromatase inhibitors in the adjuvant setting. Journal of Clinical Oncology 21 2597-2599.

Yager JD \& Davidson NE 2006 Estrogen carcinogenesis in breast cancer. New England Journal of Medicine 354 270-282.

Yue W, Santen RJ, Wang JP, Li Y, Verderame MF, Bocchinfuso WP, Korach KS, Devanesan P, Todorovic R, Rogan EG et al. 2003 Genotoxic metabolites of estradiol in breast: potential mechanism of estradiol induced carcinogenesis. Journal of Steroid Biochemistry and Molecular Biology $\mathbf{8 6}$ 477-486. 